\title{
Rippling and a phase-transforming mesoscopic model for multiwalled carbon nanotubes
}

\author{
M. Arroyo* I. Arias \\ Department of Applied Mathematics 3, LaCàN \\ Universitat Politècnica de Catalunya (UPC), Barcelona 08034, Spain
}

\begin{abstract}
We propose to model thick multiwalled carbon nanotubes as beams with nonconvex curvature energy. Such models develop stressed phase mixtures composed of smoothly bent sections and rippled sections. This model is motivated by experimental observations and large-scale atomistic-based simulations. The model is analyzed, validated against large-scale simulations, and exercised in examples of interest. It is shown that modelling MWCNTs as linear elastic beams can result in poor approximations that overestimate the elastic restoring force considerably, particularly for thick tubes. In contrast, the proposed model produces very accurate predictions both of the restoring force and of the phase pattern. The size effect in the bending response of MWCNTs is also discussed.
\end{abstract}

Key words: A. buckling, A. phase transformation, B. beams and columns, B. finite strain, B. multiwalled carbon nanotubes

\section{Introduction}

Since their discovery in 1991 (Iijima, 1991), carbon nanotubes (CNTs) have attracted much attention because of a unique combination of properties. A very special crystallographic and geometric structure, often nearly flawless, results in remarkable mechanical, electronic, thermal and chemical properties. Mechanics plays a very important role, not only because arguably CNTs are the stiffest and strongest material in Nature, but also because the electronic and chemical properties are a strong function of deformation (Tombler

\footnotetext{
* Corresponding author

Email addresses: marino.arroyo@upc.edu (M. Arroyo), irene.arias@upc.edu (I. Arias).
} 
et al., 2000; Srivastava et al., 1999). It is not surprising that CNTs appear as a fundamental building-block in an array of nanostructured multifunctional materials and nanoscale devices (see Tombler et al. (2000); Papadakis et al. (2004); Poncharal et al. (1999); Zhang et al. (2004); Cao et al. (2005); Lau et al. (2003) to name a few that are referenced elsewhere in the paper).

The focus of the present paper is on the mechanics of multiwalled carbon nanotubes (MWCNTs). MWCNTs can be geometrically understood as multiple layers of graphene rolled into nested cylinders. The theoretical study of the mechanics of CNTs has been a very active topic of research in the recent years, ranging from their elastic properties (Kudin et al., 2001; Sánchez-Portal et al., 1999) to the study of how they break through plasticity or brittle fracture (Dumitrica et al., 2006). In the study of fracture, the role of defects has been highlighted by several studies (Mielke et al., 2004; Zhang et al., 2005; Thare et a1., 2007) to harmonize theory and fracture experiments on individual MWCNTs (Yu et al., 2000). Although the linearized elasticity moduli and the failure mechanisms are of importance, the nonlinear elasticity of CNTs is the key mechanical phenomenon in many situations of scientific and technological interest. Indeed, CNTs have shown to be extremely flexible, resilient to severe and cyclic mechanical deformation, and very prone to mechanical buckling. They have been shown to undergo dramatic geometric
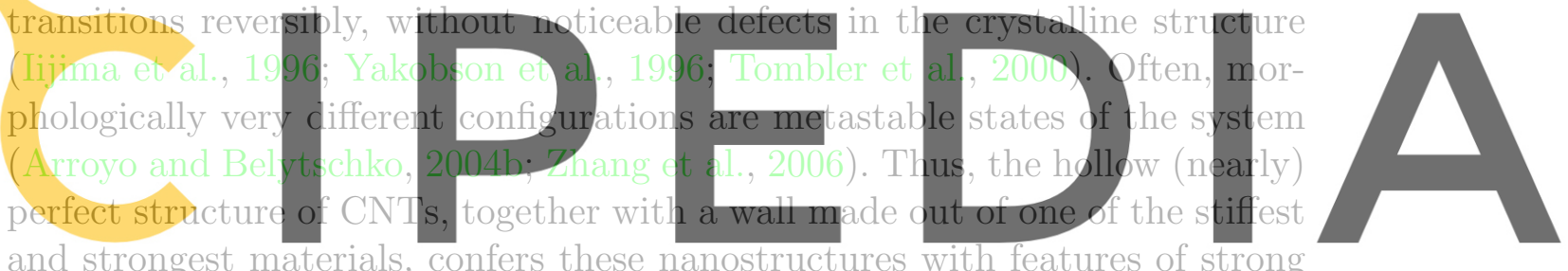

and strongest materials, confers these nanostructures with features of strong

Register for free at htt.ps/f.wwW.scipedia.com to download the version without the watermark features of flexible macromolecules.

Because of these mechanical properties, CNTs and in particular MWCNTs act as structural members in a number of devices and nanostructured materials (Lau et al., 2003; Tombler et al., 2000; Papadakis et al., 2004; Poncharal et al., 1999). In some instances, not only their geometry, high stiffness or strength is sought, but specifically the resilience and ability to undergo large deformations is exploited (Zhang et al., 2004; Cao et al., 2005). Despite the fact that in the literature the nonlinear elasticity of CNTs is well established and has been shown to have far-reaching consequences (Yakobson et al., 1996; Arroyo and Belytschko, 2003; Pantano et al., 2004), in obtaining properties of CNTs from experimental observations or in analyzing CNT-based devices, researchers predominantly use linear models of elasticity such as the Euler-Bernouilli beam theory. It is often the case that observations of anomalous mechanical behavior highlight the limitations of such models (Poncharal et al., 1999). The need for a tractable model amenable to simple analytical calculations, which nevertheless captures the complex mechanics of thick MWCNTs, is precisely 
the motivation of the present work. It is not reasonable to require large-scale atomistic or multiscale simulations on supercomputers to interpret correctly routine material characterization experiments. We show in this paper that in very common situations the usual linear elastic models provide very poor predictions, and that a slightly richer model is sufficient for an accurate description of the mechanics of thick MWCNTs.

The model we present here strongly relies on the observation that thick MWCNTs are very prone to developing rippling deformations when probed in bending. By thick we mean tubes with tens of walls or more, whose inner hollow space is much smaller than the thickness of the multi-layer structure; such nanotubes are very common as shown by the references elsewhere in the paper. As revealed by recent computations (Arroyo and Belytschko, 2003), the rippling deformations cause an anomalous anharmonic elastic behvior of the nanotubes seen as a structural member (see Section 2). This anharmonic elastic response is strongly size-dependent, as elaborated in Section 2. Based on these observations, we propose in Section 3 a mesoscopic nonlinear beam model to describe thick NWCNTS. This simple yet nontrivial model retains the es-
sential nonlinear mechanics of thick multiwalled carbon nanotubes, and allows
us to model accurately large systems with hundreds of millions of atorns. In
Section 4 we test the model agrinst large-scale simulations, and find an ex-
cellent agreement. In Section 5 we exercise the model in simple applications
illustrating the ability of the proposed model to describe the mechanics of

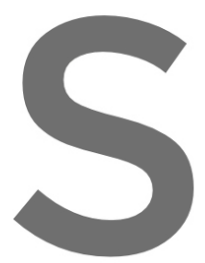
4 illustrating the ability of the proposed model to describe the mechanics of

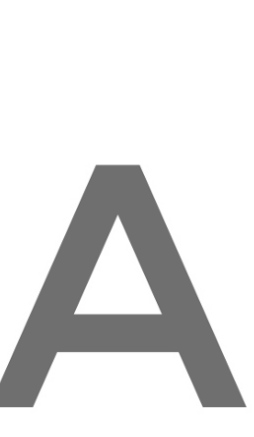

Register for free at.https//WWW.Scipedia.com to downingad the version without the watermark sible with the proposed model, but are out of reach of atomistic or even typical coarse-grained simulations.

The kind of phase-transforming beam model we propose for nanotubes was studied mathematically by James (1981). Our work is also conceptually related to other approaches to mesoscopic models for the mechanics carbon nanotubes in different regimes. For instance, Zhigilei et al. (2005) developed a model reminiscent of a discretized rod model with quadratic contributions to the strain energy, and applied it to study the dynamics of CNTs. Buehler et al. (2004) identified a regime for very long tubes in which the entropic contributions to the free energy dominate the internal energy contributions. This observation motivated the entropic model in Buehler (2006) for very long tubes. In the regime considered here, entropic effects are negligible. 


\section{Rippling of carbon nanotubes}

\subsection{Experimental record and previous theoretical work}

Rippling deformations of thick MWCNTs have been reported in the literature, see Fig. 1 for an illustration. In these TEM images, a number of MWCNTs are bent through kinematic constraints due to polymeric matrices as in Fig. 1(b-e) or to electrostatic forces as in Fig. 1(f). While single-walled CNTs or MWCNTs with a large hollow internal space typically form localized sharp kinks when subject to bending (see for instance Iijima et al. (1996) or the lower left picture in Fig. 1(b)), the absence of this internal space prevents thick MWCNTs from developing deep buckles capable of absorbing alone the deformation, and rather form sequences of morphologically well defined ripples extending over long sections of the nanotubes. A dramatic reduction in the effective Young's modulus of MWCNTs with increasing tube diameter, inferred from measured resonant frequencies and the Euler-Bernouilli linear elastic beam theory, has been attributed to the emergence of this rippling deformation mode (Poncharal It is interesting to note from the TEM micrographs in Fig. 1 that even if the deformation is not completely localized in a very narrow region as in bent SWCNTs, these MWCNTs display mixtures of rippled highly curved

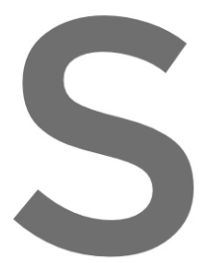
in bent SWCN s, these
regions with smoothly
the deformation is also
emphasized that these
phenomenon (B coverable rippling is follcwe
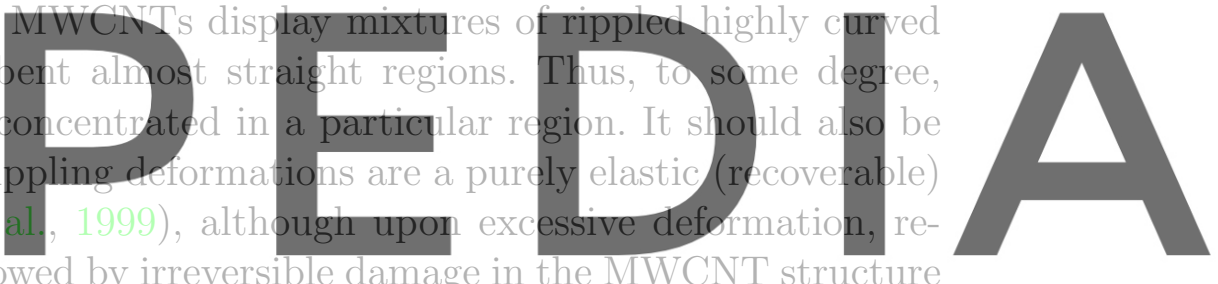

Register for free at https//www.scipedia.com to download the version without the watermark

The rippling deformations have been the subject of a number of theoretical investigations, most dealing with the understanding of the morphology of the ripples and how their wavelength and amplitude scales with the imposed curvature and the nanotube diameter. Given the practical difficulty of accessing the thick MWCNTs mechanics by direct atomistic calculations (they typically contain tens to hundreds of million atoms), different continuum approximations have been employed. In Pantano et al. (2003, 2004), elastic thin shell models of MWCNTs were treated computationally, while in Mahadevan et al. (2004) analytical calculations were performed on thin shell models inspired by experimental observations of bent rubber scrolls. This reference hypothesized that the available TEM micrographs of rippling were a manifestation of popliteal rippling observed in the rubber scrolls, while detailed computations such as those in the present paper suggest that the observed rippling in MWCNTs results from a diamond buckling pattern. As a matter of fact, for rubber scrolls loosely packed, the diamond pattern is also observed (Mahadevan, 2004). Liu et al. (2003) based their study in 2D simulations with a highly 
anisotropic nonlinearly elastic model which displayed undulations reminiscent of the rippling deformations of MWCNTs. The main goal of this reference was to explain the reduction in the effective Young's modulus with increasing diameter observed by Poncharal et al. (1999), and to this end, an elastic beam model with a scale-invariant bilinear moment-curvature relationship was proposed and exercised. The model we present here is related to this approach, but with important differences as it will become apparent later.

\subsection{Simulation method and setup}

Using finite element simulations based on an atomistic-based finite deformation continuum theory for single-layer crystalline films, it has been possible to perform reliable accurate simulations of multimillion MWCNT systems displaying rippling (see Arroyo and Belytschko (2002) for the theory and Arroyo and Belytschko (2004b) for the implementation). These computations have revealed that the diamond or Yoshimura buckling pattern is the threedimensional structure responsible for the TEM micrographs of rippling deformations, and that an anharmonic elastic response governs the mechanics of thick multiwalled carbon nanotubes (MWCNTs). While it is true that many of
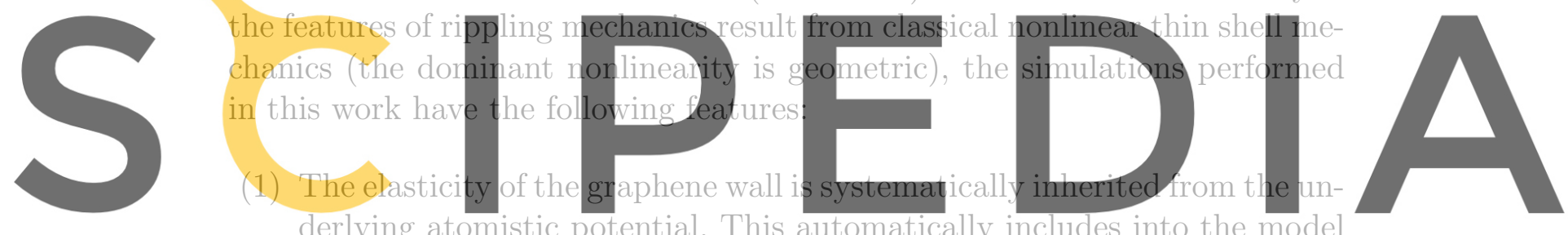

derlying atomistic potential. This automatically includes into the model

he watermark

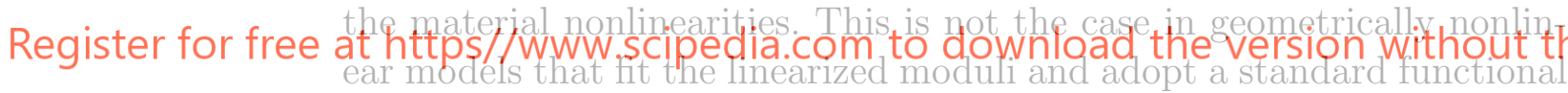
form of the strain energy for finite deformations. It has been shown in Arroyo and Belytschko (2005) that a finite deformation Kirchhoff-Saint Venant model with correct infinitesimal moduli captures accurately the small strain response of CNTs, and describes only qualitatively the large deformation mechanics. Here, the widely used Brenner potential describes for the bonded interactions (Brenner, 1990).

(2) The continuum theory provides a continuum version of the inter-wall van der Waals interactions, which replaces the double sum over the atoms by a double integral over the nanotube surface. These integrals are then approximated through numerical quadrature in the finite element implementation (Arroyo and Belytschko, 2004b). These interactions are crucial in CNT mechanics. A standard Lennard-Jones potential models the van der Waals interactions (Girifalco et al., 2000). This potential produces very smooth and weak inter-wall tangential interactions, in agreement with experimental observations (Cumings and Zettl, 2000). Upon irradiation (Kis et al., 2004) or cycling in the presence of impurities (Williams 
et al., 2002), stronger tangential inter-wall forces have been reported. This effect is ignored here.

(3) The continuum model does not require the artificial concept of a wall thickness for a two-dimensional arrangement of atoms governed by a model that views them as points (i.e. relying on the Born-Oppenheimer hypothesis). The issue of the thickness of graphene has stirred a somewhat sterile controversy in the physics and the engineering literature, the essence of which is a matter of definition and a consequence of an unnatural model. Viewing the graphene sheet as an object with thickness requires an artificial modification of the classical thin shell theory, introducing different fictitious thicknesses to produce a theory coherent with the moduli of CNTs obtained either from experiments or from ab initio calculations (see Huang et al. (2006) and references therein). Our model agrees with the view expressed for instance in Hernández et al. (1998); Kuclin et al. (2001) that the elasticity of graphene is properly described in terms of surface objects rather than bulk objects. In

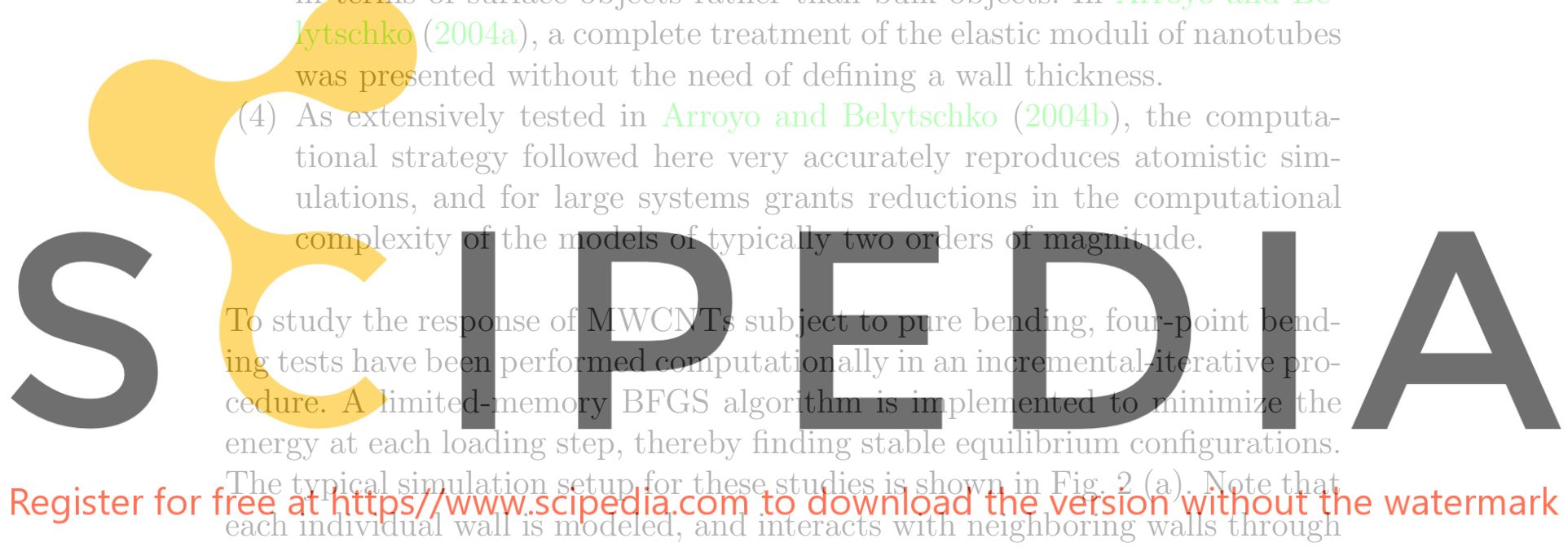

a Lennard-Jones model of the van der Waals energy. We assume that there are no other stronger coupling forces, such as those occurring in irradiated nanotubes (Huhtala et al., 2004; Kis et al., 2004), and therefore, the sliding forces between walls are very weak in agreement with ample experimental evidence. The study of the influence of stronger inter-wall coupling forces on the overall structural response of MWCNTs is certainly an interesting topic, but falls beyond the scope of the present work.

When studying the strain energy vs. curvature relation, only the central section of the tube is considered, sufficiently far away from the supports. This part of the model displays a uniform deformation state to a very high degree as checked in the post-processing of the simulations. The relevant section in the computation of the energy and the actual radius of curvature is also shown in Fig. 2 (b). The largest model considered here is a 40-walled CNT $480 \mathrm{~nm}$ long (see Fig. 3). This system contains about 31 million atoms. The reduced finite element model has only slightly over 400,000 nodes. Direct atomistic simula- 
tion of such a system is extremely challenging, while the reduction granted by the atomistic-based continuum model makes it possible to conduct systematic simulation campaigns like the one presented here. However, the computational cost of the reduced model is still quite large; on the one hand, the system is very nonlinear and thus requires an adequate number of loading steps and iterations for proper convergence. On the other hand, as detailed in Arroyo and Belytschko (2004b), even if large finite elements encompassing many atoms discretize each wall, the scale of the van der Waals interactions (the equilibrium spacing of the walls is about $0.34 \mathrm{~nm}$ ) needs to be resolved. This results in a large number of quadrature points in each finite element to integrate accurately the van der Waals interactions. For instance, the 40-walled CNT model described above required 10 million van der Waals quadrature points. Note that these are not degrees of freedom, and that the constitutive relation is evaluated in fewer quadrature points. The simulations were performed in a large-scale computing facility using up to 512 processors. The results reported are very robust with respect to changes in the size of the loading steps, the numerical tolerances, and the refinement of the finite element mesh.

The simulation setup described above is computationally expensive since only one half of the computed model is actually considered to extract the energycurvature relation. However, we have found that simpler boundary condi-
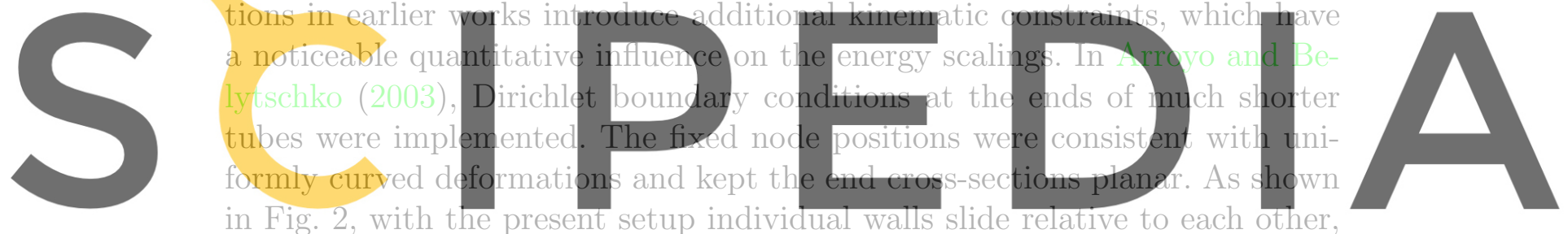

in Fig. 2, with the present setup individual walls slide relative to each other

Register for free at https, www.scipedia.com to download the version witho wit the watermark

the inner walls. Of course, depending on the actual conditions in a particular experimental setting, different constraints may become appropriate. We have chosen the four-point bending setup because it provides a clean method to probe pure bending, and it avoids unnatural or hidden constraints. We should also mention that the boundary conditions in previous studies are quite difficult to properly implement since, once rippling occurs, the neutral fiber of the MWCNT ceases to be the geometrical central fiber. The location of this neutral fiber is required to impose boundary conditions that result in a homogeneous bending state, but it is not known a priori.

\subsection{Rippling mechanics and energy scaling for thick MWCNTs}

A number of MWCNTs of different sizes (from 10 to 40 walls) have been studied. In all cases we observe that for small imposed curvatures the strain 
energy of the system (which includes the bonding energy of each graphene layer and their interaction van der Waals energy) scales harmonically, i.e.

$$
E \propto \kappa^{2}
$$

where $\kappa$ denotes the imposed curvature. The harmonic regime results from a harmonic strain energy density $w$ (strain energy per unit undeformed length of the nanotube) of the form

$$
w=\frac{1}{2} B \kappa^{2} .
$$

where the coefficient of proportionality can be very well estimated analytically as

$$
B=Y_{s} \pi \sum_{i=1}^{n} r_{i}^{3}
$$

where $Y_{s}$ is the surface Young's modulus of graphene (Hemández et al., 1998; Kudin et al., 2001; Arroyo and Belytschko, 2004a), $n$ is the number of tubes in the MWCNT and $r_{i}$ is the radius of each individual tube. We systematically observe that beyond a given curvature and coinciding with the emergence of the rippling mode, the scaling of the strain energy with deformation dramatically changes its nature to a robust power-law
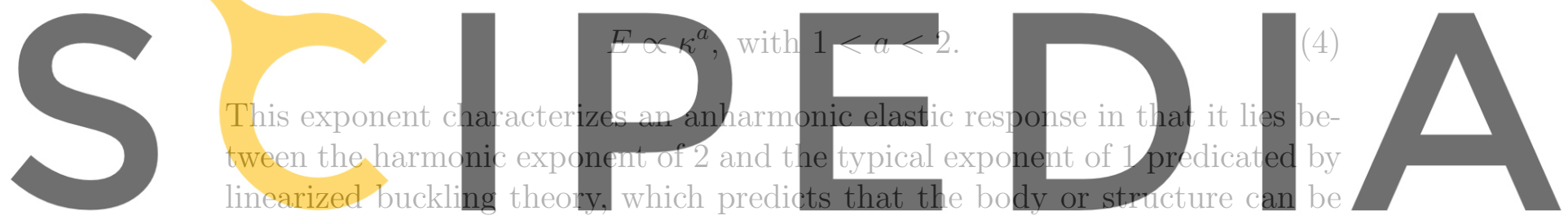

deformed at constant force. The anharmonic scaling arises from a complex bal-

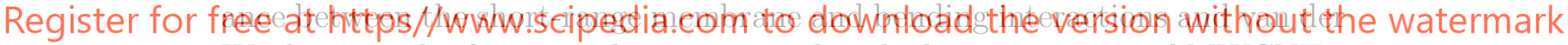
Waals intertube forces in the constrained multi-layer structure of MWCNTs.

This regime manifests itself in deformations reminiscent of the Yoshimura or diamond pattern, instead of the classical Fourier buckling modes (Bažant and Cedolin, 1991).

The transition between the harmonic and the anharmonic elastic power-laws is better represented in a logarithmic scale as shown in Fig. 2 (d). From the presented data, it is clear that the anharmonic regime emerges at moderate curvatures, and as discussed below, the thicker the nanotube the narrower the harmonic regime is. Thus, for thick tubes the bending response is fundamentally dictated by the rippling regime. To our knowledge, it has not been possible to derive analytical estimates of either the exponent $a$ or the constant of proportionality in the anharmonic power-law. Nonlinear analysis has been successful in other related situations involving thin shell crumpling (Cerda et al., 1999).

Since we are considering uniformly bent nanotubes, the anharmonic energy 
Table 1

Material parameters obtained computationally for several MWCNTs.

\begin{tabular}{lcccc} 
& $b(\mathrm{~nm})$ & $B(\mathrm{aJ} \cdot \mathrm{nm})$ & $\bar{C}(\mathrm{aJ} / \mathrm{nm})$ & $a$ \\
\hline 10-Walled CNT & 7 & $9.3 \cdot 10^{4}$ & $2.0 \cdot 10^{2}$ & 1.42 \\
20-Walled CNT & 14 & $1.4 \cdot 10^{6}$ & $4.5 \cdot 10^{2}$ & 1.42 \\
30-Walled CNT & 21 & $6.7 \cdot 10^{6}$ & $8.5 \cdot 10^{2}$ & 1.42 \\
40-Walled CNT & 28 & $2.1 \cdot 10^{7}$ & $1.2 \cdot 10^{3}$ & 1.42 \\
\hline
\end{tabular}

scaling arises from a strain energy density of the form

$$
w=C \kappa^{a}=\bar{C} \bar{\kappa}^{a}, \text { with } 1<a<2 .
$$

where the non-dimensional measure of curvature $\bar{\kappa}=b \kappa$ has been introduced and $b$ denotes the diameter of the nanotube. Therefore $\bar{C}$ has units of energy per unit length. The values for the parameters $B, \bar{C}$ and a obtained from the simulations are reported in Table 1 . These values correspond to the Brenner potential (Brenner, 1990) used in the present study, a standard analytical potential for hydrocarbon systems. It is well-known that this potential produces excessively compliant models and it has been slightly modified to produce correct elastic moduli (Arroyo and Belytschko, 2004a). The modified Brenner
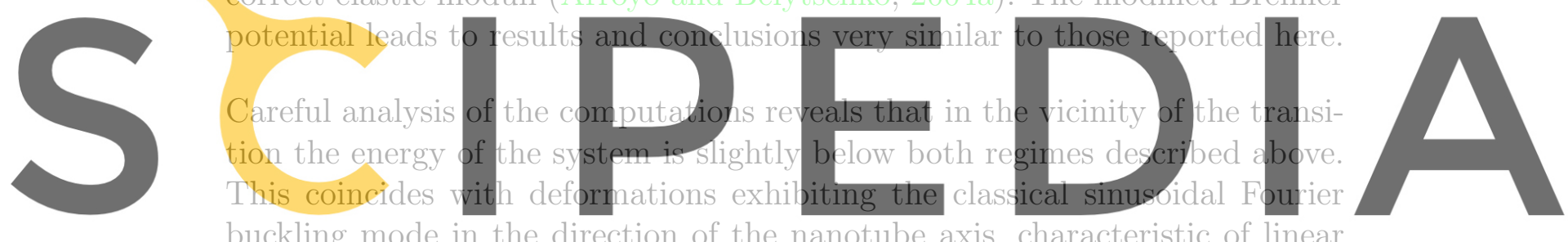

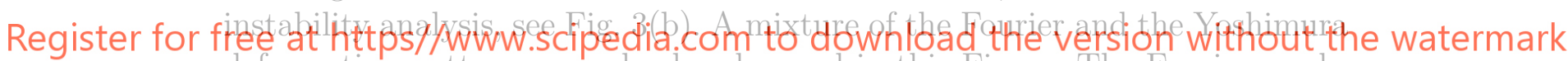
deformation patterns can also be observed in this Figure. The Fourier mechanism very early becomes less favorable than the Yoshimura pattern. Thus, the numerical results suggest that the Fourier deformation mode is the most efficient one in a very narrow range, and allows the system to tunnel between smoothly bent harmonic states and rippled Yoshimura states.

It should be pointed out that rippling deformations not only appear in bending. Torsional rippling has been also theoretically predicted (Arroyo and Belytschko, 2003, 2005), and there seems to be experimental evidence supporting this prediction (Forró and Schonenberger, 2001). In principle, it is conceivable that compressive rippling could occur. However, it is unlikely to be observed except for very short tubes since a compressed MWCNT is prone to relax through beam buckling rather than through local compressive buckling. As a matter of fact, strongly compressed and laterally constrained MWCNTs have been observed to develop compressive crushing bands (Lourie et al., 1998). In the present paper we focus on bending rippling, avoiding the complicated issue of bending-torsion-compression coupling. 


\subsection{Size effect}

We now discuss the size effect in the mechanical response of MWCNTs. To highlight the absence of scale-invariance, we first review the scale-free harmonic case. The harmonic (though geometrically nonlinear) response of an elastic rod subject to bending is characterized by a quadric growth of the strain energy density $w$ (energy per unit length of the rod) in terms of the curvature $\kappa$ of the rod:

$$
w=\frac{1}{2} Y I \kappa^{2}
$$

where $I$ denotes the moment of inertia of the cross section, and $Y$ the material's Young's modulus. The cross section of the beam is characterized by a dimension $b$ (e.g. the outer diameter of a MWCNT), and its length is denoted by $\ell$. Consider now a beam scaled by a factor $\lambda$, with $b_{\lambda}=\lambda b, \ell_{\lambda}=\lambda \ell$, $I_{\lambda}=\lambda^{4} I$, while $Y$ does not change. Consequently, the energy of the original beam and the scaled beam subject to a given non-dimensional curvature $(\bar{\kappa}=b \kappa)$ follow the relation

$$
E(\bar{\kappa})=\frac{1}{2} Y I \frac{\ell}{b^{2}} \bar{\kappa}^{2}=\lambda^{-3} \frac{1}{2} Y I_{\lambda} \frac{\ell_{\lambda}}{b_{\lambda}^{2}} \bar{\kappa}^{2}=\lambda^{-3} E_{\lambda}(\bar{\kappa})
$$
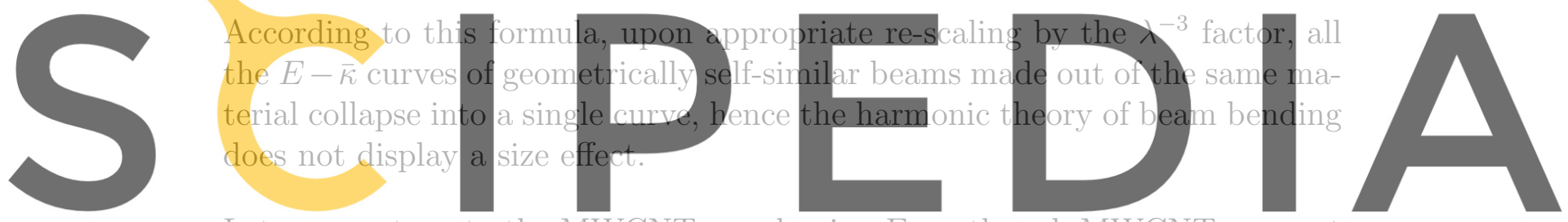

Let us now turn to the MWCNTs mechanics. Even though MWCNTs are not

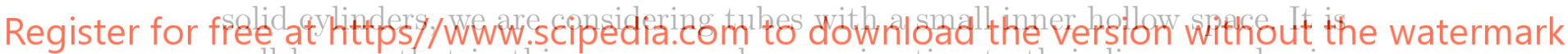

well known that in this case a good approximation to their linear mechanics is obtained by modelling thick MWCNTs as bulk cylinders with an equivalent

Young's modulus $Y=Y_{s} / t$, where $t=0.34 \mathrm{~nm}$ is the spacing between the walls and $Y_{s}$ is the surface Young's modulus of the graphene layer viewed as a continuum without thickness. We check that in the harmonic regime, the energy-curvature relationships of a 10-walled, a 20-walled, a 30-walled, and a 40-walled CNTs tested computationally indeed collapse into a single curve upon appropriate scaling. This is shown by the collapse of the blue curves in Fig. 4 (a). The energy-curvature relations have been plotted in a log-log scale, which is better suited for our purposes. However, it is apparent in this Figure that in the anharmonic regime the rescaled energy-curvature curves do not collapse, but rather show a distinct size effect. This size-dependent mechanical response is not surprising noting that the morphology of the ripples is not scale invariant, as shown in Fig. 4 (b). Specifically, we observe that:

(1) For thicker MWCNTs, the transition between the harmonic and the anharmonic regimes occurs at a smaller non-dimensional curvature. 
(2) The exponent characterizing of the anharmonic regime does not show a dependence on the size of the MWCNTs; in all cases, we find $a=1.42$.

The first observation is consistent with the fact observed in computations that for thick MWCNTs the harmonic regime ceases to be stable for very small imposed non-dimensional curvatures. As for the second observation, we note that in previous simulations (Arroyo and Belytschko, 2003) using the over-constrained Dirichlet boundary conditions described in Section 2.2, larger exponents were obtained (e.g. $a=1.66$ for a 34 -walled CNT) and a dependence of the exponent $a$ with size was observed. This dependence should be considered spurious for pure bending, but is certainly meaningful in more constrained situations. We shall see the important consequences of the size effect in structural problems later in the paper. A systematic quantitative study of the size dependence can be found in Arias and Arroyo (2007).

It should be emphasized at this point that this strong size effect is in sharp contrast with the constitutive moment-curvature behavior predicated by Liu et al. (2003). On the one hand, as further emphasized in the following section, the moment-curvature we obtain is neither monotonic nor bilinear. On the other hand, Liu et al. (2003) find a scale-invariant law. This may be due to the fact that these authors consider a simplified 2D model for the MWCNTs.

\section{Phase transforming elastica}

We next propose an elastica model with a non-convex bending energy density, which yields simple stressed microstructures consisting of mixtures of high curvature and low curvature phases. We restrict our attention to the purely static case. A similar model was studied in detail by James (1981). General solutions were obtained for buckling, and in particular is was shown that such a model produces deformations with continuous tangents and discontinuous curvatures at the phase boundaries. This model was later exercised to interpret the experimental results for polycrystalline wires made out of a shape memory alloy (Berg, 1995a,b). In three and four point bending experiments, the coexistence of low and high strain phases was observed upon stress.

More recently, Purohit and Bhattacharya (2003) and Purohit and Bhattacharya (2002) have considered beams which are non-convex in shear and stretch, but convex in curvature. It has been argued that this model is well suited for beams made out of shape memory alloy single-crystals. In contrast with the previous model, this model develops discontinuities in the tangents. The experimental TEM micrographs of MWCNTs (see Fig. 1) show mixtures of rippled highcurvature sections and smoothly bent low-curvature sections with continuity of the tangents, suggesting a model in the spirit of James (1981) in the present 
setting. As a matter of fact, the simulations presented in the previous sections suggest that indeed MWCNTs exhibit a non-convex bending energy.

\subsection{Elastic beam with non-convex curvature energy}

We consider a bending strain energy per unit reference length of the form

$$
w(\kappa)=\min \left\{\frac{1}{2} B \kappa^{2} ; C \kappa^{a}\right\},
$$

where the bending stiffness $B$ can be estimated analytically, see Eq. (3), but the parameters $C$ and $a$ are obtained from the simulations. This bending energy is supplemented by the inextensibility constraint (pure elastica) or by a stretching energy. Note that this model ignores the observation made before that in the vicinity of the transition between the harmonic and the anharmonic regimes the system lowers these two energies through a Fourier deformation mode.

It is well known (Abeyaratne and Knowles, 2006) that such a non-convex energy can lead to stressed phase mixtures. Indeed, upon the action of a bending moment $M$, the potential energy density of the system becomes

$$
P(\kappa, M)=w(\kappa)-M \kappa \text {. }
$$

The bias introduced by the loading first produces the emergence of a new local energy minimum, and hence a new high-strain phase is possible (see Fig. 5). We call $M_{m}$ the bending moment at which the energy well in the high-strain phase is created. In the present setting, the low-strain phase $L$ corresponds to the smoothly bent phase characterized by the quadratic scaling, while the high-strain phase $H$ is a rippled phase displaying the anharmonic scaling. The applied moment at which the energy levels of the two energy minima coincide is called the Maxwell moment $M_{0}$. Further applied moment eventually leads to the destruction of the low-strain minimum, for $M_{M}$. Thus, for $M_{m} \leq M \leq M_{M}$ both the smoothly bent and the rippled phases can exist. This local or material picture is complicated when a given structure is studied; for instance a macroscopic strain can in general be accommodated by nonhomogeneous states displaying mixtures of smooth and rippled deformations.

Specializing the theory in Abeyaratne and Knowles (2006) to the present setting, the necessary condition for the existence of an energy well in either phase is

$$
\frac{\partial}{\partial \kappa} P(\kappa, M)=0
$$


which provides two candidate minima characterized by the relations

$$
\kappa_{L}(M)=\frac{M}{B} \quad \text { and } \quad \kappa_{H}(M)=\left(\frac{M}{a C}\right)^{\frac{1}{a-1}}
$$

To assess whether these candidates for energy wells are indeed possible, we define the curvature at which the harmonic and the anharmonic regimes meet

$$
\kappa_{d}=\left(\frac{2 C}{B}\right)^{\frac{1}{2-a}}
$$

The low-strain phase is possible if and only if $\kappa_{L} \leq \kappa_{d}$, which leads to

$$
M \leq B\left(\frac{2 C}{B}\right)^{\frac{1}{2-a}}=M_{M}
$$

On the other hand, the high-strain phase is possible if and only if $\kappa_{H} \geq \kappa_{d}$, hence

$$
M \geq a C\left(\frac{2 C}{B}\right)^{\frac{a-1}{2-a}}=M_{m} .
$$

To determine the Maxwell bending moment, we equate the low-strain and high-strain potential energy densities

$$
P_{L}(M)=P\left(\kappa_{L}(M), M\right)=P\left(\kappa_{H}(M), M\right)=P_{H}(M),
$$

to obtain

$$
M_{0}=\left[\frac{a}{2 B(a-1)}(a C)^{\frac{1}{a-1}}\right]^{\frac{a-1}{2-a}} .
$$

For an applied moment $M$ in the range $\left[M_{m}, M_{M}\right]$ two phases are possible, and in general coexist.

At a phase boundary, the transformation curvature is defined by

$$
\kappa_{T}(M)=\kappa_{H}(M)-\kappa_{L}(M)
$$

In phase-transforming materials, besides the mechanical forces one must account for the phase or configurational forces, which are energetically conjugate to the position of the phase boundaries. Here, the configurational or driving force on an phase boundary $f$ may be understood as the energy release rate as the phase boundary moves, and can be shown to be the jump in the potential energy density at the phase boundary or equivalently an integral of the transformation curvature:

$$
f=\llbracket P(\kappa, M) \rrbracket=\int_{M_{0}}^{M} \kappa_{T}\left(M^{\prime}\right) d M^{\prime}
$$

Since a phase boundary can only exist if the bending moment lies in the interval $\left[M_{m}, M_{M}\right]$, it follows that the driving force on the phase boundary 
lies in the interval $\left[f_{m}, f_{M}\right]$ where

$$
f_{m}=P_{L}\left(M_{m}\right)-P_{H}\left(M_{m}\right)=\int_{M_{0}}^{M_{m}} \kappa_{T}\left(M^{\prime}\right) d M^{\prime}<0
$$

and

$$
f_{M}=P_{L}\left(M_{M}\right)-P_{H}\left(M_{M}\right)=\int_{M_{0}}^{M_{M}} \kappa_{T}\left(M^{\prime}\right) d M^{\prime}>0
$$

From these expressions it is clear that the diving force acting on an phase boundary vanishes if the moment in the elastica coincides with the Maxwell moment.

\subsection{Nucleation and propagation criteria}

It is well known that the equilibrium configurations of nonlinearly elastic materials with non-convex energies are not uniquely determined by the combination of the mechanical equilibrium, bulk constitutive relation and boundary conditions. Additional information about the material is needed in order to select amongst the multiple positions of a phase boundary compatible with mechanical equilibrium. A general way of removing the degeneracy of conventional continuum mechanics for phase transforming materials is to append the standard model with a nucleation criterion (given by critical stresses or driving forces for nucleating a high-strain phase from a low-strain phase $f_{n}^{L H}$ and vice-versa $f_{n}^{H L}$ ) and a kinetic law prescribing the velocity of the phase boundary $\dot{s}$ as a function of the driving force $f$ acting on it, or inversely by the relation $f=\phi(\dot{s})$. In the kinetic relation, an important parameter is the resistance of a phase boundary to its motion, expressed in terms of the driving force needed to enlarge the high-strain phase $f_{r}^{L H}$ and that needed for a motion of the phase boundary that makes the high-strain phase smaller $f_{r}^{H L}$ (see Abeyaratne and Knowles (2006) for details).

The purely static simulations of MWCNTs carried out in the present study have provided valuable information regarding the bending energetics, but do not provide sufficient data to model in detail the transition from one phase to the other and the kinetic law. Indeed, on the one hand, rate effects are absent in these simulations. On the other hand, despite we have observed a Fourier deformation mode near the transition, which possibly lowers the energetic barrier predicated by the elementary model encapsulated in Eq. (8), we have not attempted to quantify and model its effect on the nucleation and kinetics of the phase transformation. Instead, we consider two extreme but illuminating rate-independent regimes: an energetic criterion, according to which any material point adopts instantaneously the lowest energy state, and a rate-independent frictional kinetic criterion, by virtue of which the material switches from one phase to the other if and only if the energy well of the 
current phase is destroyed. Any other choice of nucleation criterion and rateindependet kinetic law falls within these two extremes.

\section{Energetic criterion}

The aforementioned degeneracy can be eliminated by requiring that each material point adopts instantaneously the state with the lowest possible energy, irrespective of the energy barrier that may be present. Physically, this can be attributed to thermal agitation or any other tunneling mechanism not included in the model. This prescription provides an extreme nucleation criterion and kinetic law. Indeed, new phases are nucleated if and only if they are energetically favorable. On the other hand, phase boundaries move in such a way that the total energy is minimized, i.e. phase equilibrium is enforced in addition to mechanical equilibrium. This amounts to requiring that at any phase boundary $f=0$, and thus, recalling Eq. (18), at the phase boundary the bending moment is $M_{0}$ and the kinetic law is simply $\phi(\dot{s})=0$.

More operationally, at any given material point subjected to a stress $M \in$ $\left[M_{m}, M_{M}\right]$, the minimum potential energy alone selects the phase of the material; if $P_{L}(M)<P_{H}(M)$ then $\kappa=\kappa_{L}(M)$, and conversely if $P_{L}(M) \geq P_{H}(M)$ then $\kappa=\kappa_{H}(M)$. For this model, the nucleation stress from the low to the high strain phase coincides with the nucleation stress from the high to the low strain phase $M_{n}^{L H}=M_{0}=M_{n}^{H L}$, and a material phase boundary can resist no driving force at all, hence the propagation driving forces needed to enlarge or decrease the high strain phase vanish, $f_{r}^{L H}=f_{r}^{H L}=0$. Obviously, for $M<M_{m}$ only the $L$ phase is possible, hence $\kappa=\kappa_{L}(M)$, and similarly for $M>M_{M}$ it follows that $\kappa=\kappa_{H}(M)$.

This energetic criterion leads to the so-called Maxwell processes. A simple Maxwell process for a uniformly stressed elastica subject to an increasing bending moment is sketched in the rightmost plot in Fig. 6 in magenta. It follows the $L$ response from the origin to its intersection with the horizontal line $M=M_{0}$. At this point, the high-strain phase nucleates at some point of the elastica, for instance at a boundary, and propagates through the material. The macroscopic moment-curvature relationship then follows the line $M=M_{0}$ until it intersects the $H$ response. After this point, the whole elastica has transformed into the high-strain phase. Upon unloading, the systems follows exactly the same path, and therefore does not exhibit any kind of hysteresis nor dissipation in such a cycle. 
As an opposite extreme case, we consider a rate-independent "frictional" kinetic law

$$
f=\phi(\dot{s})=\left\{\begin{array}{l}
f_{M} \text { if } \dot{s}>0 \\
f_{m} \text { if } \dot{s}<0
\end{array}\right.
$$

For $\dot{s}=0$, the driving force lies in the interval $\left[f_{m}, f_{M}\right]$, and is determined by mechanical equilibrium. According to this law, a given material point stays in its current phase unless this phase becomes impossible. Thus, as before for $M<M_{m}$ we have $\kappa=\kappa_{L}(M)$, and for $M>M_{M}$ it follows that $\kappa=\kappa_{H}(M)$. If $M \in\left[M_{m}, M_{M}\right]$, then the material stays in its current phase. This model adopts the extreme allowable values for the nucleation and propagation driving forces

$$
f_{r}^{L H}=f_{n}^{L H}=f_{M} \quad \text { and } \quad f_{r}^{H L}=f_{n}^{H L}=f_{m} .
$$

Physically, this kinetic law and nucleation criterion assumes that the system cannot overcome the energy barrier between states, no matter how low it is. Such a model produces hysteretic loading-unloading cycles, as illustrated in the rightmost plot of Fig. 6 in green.

\subsection{Cantilever beam and three-point-bending solutions}

We now review simple boundary value problems with non-homogeneous stress states for the geometrically exact phase-transforming elastica model. We consider a cantilever beam of length $L$ described by $x \in[0, L]$ and $y=0$ in its undeformed configuration. The leftmost point of the beam is clamped, while a vertical force $(0, F)$ is applied on the beam at a fixed value $x=\ell<L$ irrespective of the deformation of the beam. Note that this is not a follower load (such as the one that would act on the tip of the cantilever following this material point through the deformation), which simplifies the problem. We consider a Monge parametrization of the deformed beam $y(x)$. The arc-length parameter labels material particles, and it is given by $s=\int_{0}^{x} \sqrt{1+\left(y^{\prime}(\xi)\right)^{2}} d \xi$. We assume that $L$ is large enough so that throughout the deformation the abscissa of the end point of the beam satisfies $x_{\text {end }}>\ell$. The bending moment on the elastica is $M(x)=(\ell-x) F$ for $x<\ell$ and zero otherwise. Thus, in a loading path starting from $F=0$ the high strain phase will nucleate at the clamped end irrespective of the nucleation criterion, and the phase boundary will never reach the point where the load is applied, no matter how large $F$ is. At each point in the elastica the bending moment is known and therefore either the energetic or the frictional criteria given above provide a prescription for the corresponding curvature $\kappa(x)$ (the latter requires in addition keeping track of the state of the material point). Finally, recalling the expression of the curvature of a curve described by the Monge parametrization, we can integrate 
$y(x)$ from the ODE

$$
y^{\prime \prime}(x)-\kappa(x)\left[1+\left(y^{\prime}(x)\right)^{2}\right]^{\frac{3}{2}}=0, \quad y(0)=0, \quad y^{\prime}(0)=0 .
$$

This ODE is integrated for $x \in(0, \ell)$ since the part of the cantilever beam beyond the point of application of the load has zero bending moment and is rectilinear. To consider a three-point-bending test with a constant distance between the end supports $2 \ell$ and an applied force in the central support of $2 F$, in which the elastica can slide on the end supports upon deformation (see Berg (1995b) for an experimental setup), it is sufficient to complete the above solution by the symmetry $y(-x)=y(x)$.

By way of illustration, Fig. 7 shows a loading-unloading cycle for an elastica model for a 20-walled CNT with the parameters $C$ and $a$ extracted from the large scale simulations. In this example, $\ell=112 \mathrm{~nm}$. Both the reversible energetic and the hysteretic frictional models have been considered. It can be noticed that despite the peak reaction force is almost identical in both cases, the extent of the high-strain rippled phase for the energetic model is considerably larger.

\section{Comparison between the large-scale simulations and the phase- transforming elastica}

To assess the ability of the phase-transforming elastica to describe the mechanics of MWCNTs, we consider a simple benchmark example with a nonuniform stress state along the tube: a three-point-bending test. We compare the results given by large-scale three-dimensional simulations with the method described in Section 2.2 performed in a supercomputer with those obtained analytically from the elastica model. The parameters $C$ and $a$ are extracted from the simulations of MWCNTs under uniform bending described in Section 2 , and should be viewed in the present context as material parameters. The objective of the comparison is twofold: it serves as a validation of the mesoscopic phase-transforming elastica and it interrogates the full system about the appropriateness of the considered phase-boundary kinetic laws.

We consider a 20-walled CNT $280 \mathrm{~nm}$ long, which contains some 4.7 million atoms. The computational model in these simulations has around 100,000 finite element nodes. The simulation setup and typical deformations are shown in Fig. 8. The simulations are driven by the relative motion of the supports in the direction normal to the initially straight MWCNT. It can be observed that rippling starts to develop in the vicinity of the central support, where the bending moment is maximum. The rippled region then propagates through 
the MWCNT towards the end supports as the load is increased. The diamond pattern in the rippled regions can be clearly observed.

The reaction-displacement response of the full system as given by the simulations is plotted in Fig. 9 (black dots). To get meaningful comparisons, the simulations must be carefully post-processed to factor out the flexibility of the supports, i.e. the local deformation of the outer wall at these points. To this end, the central fiber of the MWCNT is obtained in terms of the inner wall (see the black line in Fig. 10). The response as predicted by the phasetransforming elastica supplemented by the energetic (magenta) and frictional (green) criteria is also plotted. The agreement between the large-scale simulations and the simple model is remarkable. In the full model, the response deviates from linear elasticity at the point predicted by the energetic criterion, and then stays between the magenta and the green curves.

To test whether the system exhibits any kind of friction, or rather accommodates locally to the minimum energy state, we consider a closed loading cycle. We find that upon unloading, the large-scale model does not exhibit noticeable hysteresis, but rather a reversible response within the numerical tolerances (the unloading data is also represented in Fig. 9 but cannot be distinguished from the loading data). This indicates that no spurious rate effects are introduced by the incremental-iterative numerical solution method. This also suggests that the energy barrier between the harmonic phase and the anharmonic (rippled) phase is lowered by a mechanism not included in the simple energy in Eq. (8). Indeed, a careful analysis of the deformations in Fig. 8 reveals a barely noticeable Fourier deformation pattern in the vicinity of the transition between the rippled and the smoothly bent regions, which is consistent with the observation put forward in Section 2.3. Based on this evidence, we hypothesize that the system mobilizes this deformation mechanism to efficiently transition (tunnel) between the harmonic and the anharmonic energy wells.

The above discussion strongly suggests that the appropriate kinetic law in the rate-independent limit is given by the strictly energetic criterion. The slightly higher restoring force of the full system as compared to the phase transforming elastica with the energetic criterion (see Fig. 9) can be caused by a variety of phenomena ignored in our simple model. One possible explanation is that the system pays an energetic price for variations in the amplitude of the rippling deformation. Figure 8 clearly shows this variation in the strength of the Yoshimura mode when the stress state is not uniform. The manifestation of this effect in the elastica model would be a curvature (strain) gradient energy term in the anharmonic regime. Rate effects could in principle also explain the observed disagreement, but they can be excluded here since we have checked that the response is strictly reversible upon unloading. 
A further argument against the frictional kinetic law for the system under study is provided by the comparison of the phase patterns given by the full model and the two versions of the elastica model. We find that the frictional model predicts rippled phases which are considerably smaller than those observed in the tree-dimensional simulations, while the phase distributions given by the energetic criterion agree very well with the full model. Figure 10 shows a side view of the three-dimensional simulations, together with the central fiber post-processed from the 3D data. The deformation predicted by the phasetransforming purely energetic elastica model is super-imposed in this plot and color-coded (red for the high-curvature phase, blue for the low-curvature phase). The agreement in the deformations and the phase-pattern at different load levels is again remarkable.

\section{Applications}

Once the ability of the phase-transforming elastica to encapsulate the complex mechanics of rippling has been established, we exercise the model in applications of interest.

\subsection{Size effect in MWNCTs subject to three point bending}

We first investigate the consequences in non-uniform bending situations of the size effect of the constitutive response of MWCNTs reported Section 2.4. To this end, we consider three geometrically self-similar three-point-bending tests for nanotubes of different diameters. Three-point-bending tests have been performed experimentally on CNTs, see Tombler et al. (2000) for an experimental setup involving a trench and an atomic force microscope. If the material response is linear elastic, then it is scale-invariant and the reaction at the central point scales as the size of the beam to the power of two. We perform the test on the phase-transforming elastica model with the material parameters of Table 1. The results are reported in Fig. 11.

It is apparent from the Fig. 11 that the thicker the MWCNT, the sooner a high-strain rippled phase appears in the center of the beam. For the 40walled CNT, the nucleation of the rippled phase occurs for a barely noticeable deformation. After nucleation, the rippled phase propagates towards the outer supports. The extent of this phase at the end of the experiment is also larger the thicker the tube is. The Figure also shows that the reaction exerted by the MWCNTs that develop rippling is only a fraction of the reaction expected from the simple linear elastic Euler-Bernouilli beam model (66\% for the 10 -walled tube, $40 \%$ for the 20 -walled tube and $28 \%$ for the 40 -walled tube, predicted 
with the energetic model). This reduction of the effective elastic modulus as the diameter is increased is consistent with the experimental results reported in Poncharal et al. (1999).

\subsection{Indentation of a forest of vertically aligned CNTs}

In recent years, a number of groups have synthesized forests of vertically aligned CNTs (see for example Lau et al. (2003)). These systems show promise in a number of applications such as super-hydrophobic surfaces, or field emitters. Their mechanical characterization has been carried out through atomic force microscopy (AFM). Qi et al. (2003) proposed a consecutive contact model to predict the force-penetration curves of a given forest, and used it to support the interpretation of experimental curves. These authors assumed that the MWCNTs behave as linearly elastic Euler-Bernouilli beams. This is a good assumption for small indentation depths, or for MWCNTs with a large aspect ratio that accommodate large tip displacements with small curvatures relative to $\kappa_{d}$.

Here we consider a simplified model of MWCNT forest in which the tubes are disposed perpendicular to a substrate in a 2D hexagonal lattice. We consider $500 \mathrm{~nm}$ long 40-walled tubes, whose diameter is around $28 \mathrm{~nm}$, and a density of 200 nanotubes per squared micron. The aspect ratio is similar to those reported in Qi et al. (2003). This forest is penetrated by a diamond-tip AFM, which is modeled as a three-sided pyramid with apex angle $\phi=60^{\circ}$. Following Qi et al. (2003), we assume that each nanotube contacts the AFM tip just at its end. For the tip considered, this hypothesis is valid for indentation depths smaller than $70 \%$ of the length of the tubes. As the tip is lowered, it successively contacts tubes that deform as cantilever beams with a force acting on their tip. Friction on the AFM tip is neglected, and consequently these forces are assumed to be normal to the tip faces.

By computing the force-displacement curve of a single tube (we consider here a follower load at the tip of the geometrically-exact phase transforming elastica with the energetic criterion), it is easy to compute the theoretical forcepenetration curve. The setup and the results are reported in Fig. 12, both for the proposed phase-transforming model and for a conventional elastic beam model. It can be observed that both models agree for small indentation depths, when the tubes closer to the apex have not reached the nucleation bending moment. For larger indentation depths, the tubes successively and progressively develop rippling and consequently the force on the indenter force drifts from that predicted by the simple beam model. The linear elastic beam model over-estimates the indentation force at the end of the test by almost $50 \%$. 


\section{Conclusions and outlook}

We have presented a mesoscopic model for the mechanics of thick MWCNTs. In this model the rippling deformations are viewed as a phase transition for a beam with non-convex bending energy. The model is suggested by experimental observations and by the results of large scale simulations using an atomistic-based continuum model discretized by finite elements (Arroyo and Belytschko, 2004b). These simulations and experiments (Poncharal et al., 1999) show that thick nanotubes are very prone to rippling. In the rippling regime, a complex balance of membrane, bending and inter-wall energies, together with the geometric structure of MWCNTs, results in an anomalous yet very robust anharmonic power-law behavior of the energy-curvature relation $E \propto \kappa^{a}$ with $1<a<2$. It is noteworthy that the reversible nonlinear atomistic mechanics of CNTs can first be described by a continuum surface model (Arroyo and Belytschko, 2002), and then the collective complex behavior of many shells can be encoded into a simple power-law. The anharmonic elastic regime is characterized in pure bending by a size dependent multiplicative factor and by a size independent exponent $a \approx 1.42$. As a result, the thicker the MWCNT, the sooner rippling develops and the more relevant the anharmonic regime is in its structural response.

Using well-known results for phase-transforming materials, the consequences of the non-convex bending energy have been studied. In particular, we have highlighted the emergence of stressed mixtures of a high-strain (rippled) phase and a low-strain (smoothly bent) phase, and the need for a nucleation criterion and a kinetic law to uniquely determine the mechanics of the phasetransforming elastica. Since the large-scale static simulations upon which we build the mesoscopic model do not provide sufficient information about the kinetics or nucleation, we have considered two extreme rate-independent cases: a purely energetic model that selects the phase of a material point solely on the basis of energy minimization, and a frictional model that forces each material point to stay in its current phase unless the corresponding energy well is destroyed.

We have compared the results provided by the phase-transfoming elastica and those provided by large-scale simulations for a 20-walled CNTs subject to three-point-bending. The comparison shows that the agreement in the forcedisplacement curve is excellent, and lies between the response provided by the energetic and the frictional versions of the phase-transforming elastica. The simulations provide two important clues that strongly support the energetic model; on the one hand, upon unloading the full 3D system does not exhibit hysteresis, and on the other hand, the deformations and phase patterns of the energetic model match very accurately those of the full model while the frictional model produces much smaller rippled phases. Based on the large- 
scale simulations, we speculate that the emergence of a Fourier deformation mode near the onset of rippling may help the system tunnel between the harmonic smoothly bent state and the Yoshimura rippled state, effectively reducing the energy barrier between the two potential energy wells.

We have shown that the mesoscopic proposed model can efficiently and accurately describe the mechanics of MWCNT-based devices and materials involving possibly large numbers of long tubes. Recent examples of such materials include foams and yarns made out of MWCNTs (Zhang et al., 2004; Cao et al., 2005). The proposed model can also be applied to study the bending dynamics of MWCNTs (Poncharal et al., 1999). However, this requires a possibly rate-dependent model for the kinetic law. The identification of such a model from large-scale dynamic simulations is currently under investigation. The results presented here pose an important limitation on the kinetic law for the phase-transforming beam: it should coincide with the energetic model in the rate-independent limit. The parameter $C$ in the proposed model has been shown to be size-dependent, and the parameter $a$ is different for torsion and bending. Unfortunately, we have not been able to obtain analytical estimates for these parameters in terms of the geometric and material properties of the MWCNTs, although a study on the systematics of these parameters with size can be found in Arias and Arroyo (2007). Such estimates would greatly facilitate the practical application of the proposed phase-transforming elastica.

\section{Acknowledgements}

The support of the European Commission through the Marie Curie Actions (MA, MIRG-CT-2005-029178; IA, MIRG-CT-2005-029158) is gratefully acknowledged. The authors thankfully acknowledge the computer resources, technical expertise and assistance provided by the Barcelona Supercomputing Center - Centro Nacional de Supercomputación.

\section{References}

Abeyaratne, R., Knowles, J., 2006. Evolution of Phase Transitions: A Continuum Theory. Cambridge University Press.

Arias, I., Arroyo, M., 2007. Size-dependent nonlinear elastic scaling of multiwalled carbon nanotubes. Submitted.

Arroyo, M., Belytschko, T., 2002. An atomistic-based finite deformation membrane for single layer crystalline films. Journal of the Mechanics and Physics of Solids 50 (9), 1941-1977.

Arroyo, M., Belytschko, T., 2003. Nonlinear mechanical response and rippling 
of thick multi-walled carbon nanotubes. Physical Review Letters 91 (21), 215505.

Arroyo, M., Belytschko, T., 2004a. Finite crystal elasticity of carbon nanotubes based on the exponential cauchy-born rule. Physical Review B 69, 115415.

Arroyo, M., Belytschko, T., 2004b. Finite element methods for the non-linear mechanics of crystalline sheets and nanotubes. International Journal for Numerical Methods in Engineering 59 (3), 419-456.

Arroyo, M., Belytschko, T., 2005. Continuum mechanics modeling and simulation of carbon nanotubes. Meccanica 40, 455-469.

Bažant, Z., Cedolin, L., 1991. Stability of structures. Oxford University Press.

Berg, B., 1995a. Bending of superelastic wires, Part I: Experimental aspects. Journal of Applied Mechanics 62, 459-465.

Berg, B., 1995b. Bending of superelastic wires, Part II: Application to threepoint bending. Journal of Applied Mechanics 62, 466-470.

Bower, C., Rosen, R., Jin, L., 1999. Deformation of carbon nanotubes in nanotube - polymer composites. Applied Physics Letters 74 (22), 3317-3319.

Brenner, D., 1990. Empirical potential for hydrocarbons for use in simulating chemical vapor deposition of diamond films. Physical Review B 42 (15), 9458-9471.

Buehler, M., 2006. Mesoscale modeling of mechanics of carbon nanotubes: Self-assembly, self-folding and fracture. Journal of Materials Research in press (69), 69-96.

Buehler, M., Kong, Y., Gao, H., 2004. Deformation mechanisms of very long single-wall carbon nanotubes subject to compressive loading. Journal of Engineering Materials and Technology 126 (3), 245-249.

Cao, A., Dickrell, P., Sawyer, W., Ghasemi-Nejhad, M., Ajayan, P., 2005. Super-compressible foamlike carbon nanotube films. Science 310, 1307-1310.

Cerda, E., Chaieb, S., Melo, F., Mahadevan, L., 1999. Conical dislocations in crumpling. Nature 401, 46-49.

Cumings, J., Zettl, A., 2000. Low-Friction Nanoscale Linear Bearing Realized from Multiwall Carbon Nanotubes. Science 289 (5479), 602-604.

Dumitrica, T., Hua, M., Yakobson, B., 2006. Symmetry-, time-, and temperature-dependent strength of carbon nanotubes. Proceedings of the National Academy of Sciences 103 (16), 6105-6109.

Forró, C., Schonenberger, C., 2001. Physical properties of multi-wall nanotubes. Topics in Applied Physics 80, 329-390.

Girifalco, L., Hodak, M., Lee, R., 2000. Carbon nanotubes, buckyballs, ropes, and a universal graphitic potential. Physical Review B 62 (19), 13104-13110.

Hernández, E., Goze, C., Bernier, P., Rubio, A., 1998. Elastic properties of $\mathrm{C}$ and $\mathrm{B}_{n} \mathrm{C}_{y} \mathrm{~N}_{z}$ composite nannotubes. Physical Review Letters 80 (20), $4502-4505$.

Huang, Y., Wu, J., Hwang, K., 2006. Thickness of graphene and single-wall carbon nanotubes. Physical Review B 74, 245413.

Huhtala, M., Krasheninnikov, A., Aittoniemi, J., Stuart, S., Nordlund, K., Kaski, K., 2004. Improved mechanical load transfer between shells of mul- 
tiwalled carbon nanotubes. Physical Review B 70, 045404.

Iijima, S., 1991. Helical microtubules of graphitic carbon. Nature 354, 56-58. Iijima, S., Brabec, C., Maiti, A., Bernholc, J., 1996. Structural flexibility of carbon nanotubes. Journal of Chemical Physics 104 (5), 2089-2092.

James, R., 1981. The equilibrium and post-buckling behavior of an elastic curve governed by a non-convex energy. Journal of Elasticity 11 (3), 239269.

Khare, R., Mielke, S. L., Paci, J. T., Zhang, S. L., Ballarini, R., Schatz, G. C., Belytschko, T., 2007. Coupled quantum mechanical/molecular mechanical modeling of the fracture of defective carbon nanotubes and graphene sheets. Physical Review B 75 (7), 075412.

Kis, A., Csányi, G., Salvetat, J., Lee, T.-N., Couteau, E., Kulik, A., Benoit, W., Brugger, J., Forró, L., 2004. Reinforcement of single-walled carbon nanotube bundles by intertube bridging. Nature Materials 3, 153-157.

Kudin, K., Scuseria, G., Yakobson, B., 2001. $\mathrm{C}_{2}$, BN, and C nanoshell elasticity from ab initio computations. Physical Review B 64, 235406.

Kuzumaki, T., Hayashi, T., Ichinose, H., Miyazawa, K., Ito, K., Ishida, Y., 1998. In-situ observed deformation of carbon nanotubes. Philosophical Magazine A 77 (6), 1461-1469.

Lau, K. K. S., Bico, J., Teo, K. B. K., Chhowalla, M., Amaratunga, G. A. J., Milne, W. I., McKinley, G. H., Gleason, K. K., Dec 2003. Superhydrophobic carbon nanotube forests. Nano Letters 3 (12), 1701-1705.

Liu, J., Zheng, Q., Jiang, Q., 2003. Effect of bending instabilities on the measurements of mechanical properties of multiwalled carbon nanotubes. Physical Review B 67, 075414.

Lourie, O., Cox, D., Wagner, H., 1998. Buckling and collapse of embedded carbon nanotubes. Physical Review Letters 81 (8), 1638-1641.

Mahadevan, L., 2004. Personal communication .

Mahadevan, L., Bico, J., McKinley, G., 2004. Popliteal rippling of layered elastic tubes and scrolls. Europhysics Letters 65 (3), 323-329.

Mielke, S., Troya, D., Zhang, S., Li, J., Xiao, S., Car, R., Ruoff, R., Schatz, G., Belytschko, T., 2004. The role of vacancy defects and holes in the fracture of carbon nanotubes. Chemical Physics Letters 390 (4-6), 413-420.

Pantano, A., Boyce, M. C., Parks, D. M., Oct 2003. Nonlinear structural mechanics based modeling of carbon nanotube deformation. Phys. Rev. Lett. 91 (14), 145504.

Pantano, A., Parks, D., Boyce, M., 2004. Mechanics of deformation of single and multi-wall carbon nanotubes. Journal of the Mechanics and Physics of Solids 52 (4), 789-821.

Papadakis, S., Hall, A., Williams, P., Vicci, L., Falvo, M., Superfine, R., S, S. W., 2004. Resonant oscillators with carbon-nanotube torsion springs. Physical Review Letters 93 (14), 146101.

Poncharal, P., Wang, Z., Ugarte, D., de Heer, W., 1999. Electrostatic deflections and electromechanical resonances of carbon nanotubes. Science 283, 1513-1516. 
Purohit, P., Bhattacharya, K., 2002. On beams made of a phase-transforming material. International Journal of Solids and Structures 39, 3907-3929.

Purohit, P., Bhattacharya, K., 2003. Dynamics of strings made of phasetransforming materials. Journal of the Mechanics and Physics of Solids 51, $393-424$.

Qi, H., Teo, K., Lau, K., Boyce, M., Milne, W., Robertson, J., Gleason, K., 2003. Determination of mechanical properties of carbon nanotubes and vertically aligned carbon nanotube forests using nanoindentation. Journal of the Mechanics and Physics of Solids 51, 2213-2237.

Sánchez-Portal, D., Artacho, E., Soler, J., Rubio, A., Ordejón, P., 1999. Ab initio structural, elastic, and vibrational properties of carbon nanotubes. Physical Review B 59 (19), 12678-12688.

Srivastava, D., Brenner, D., Schall, J., Ausman, K., Yu, M., Ruoff, R., 1999. Predictions of enhanced chemical reactivity at regions of local conformational strain on carbon nanotubes: Kinky chemistry. Journal of Physical Chemistry B 103 (21), 4330-4337.

Tombler, T., Zhou, C., Alexseyev, L., Kong, J., Dai, H., Liu, L., Jayanthi, C., Tang, M., Wu, S., 2000. Reversible electromechanical characteristics of carbon nanotubes under local-probe manipulation. Nature 405, 769 - 772.

Williams, P., Papadakis, S., Patel, A., Falvo, M., Washburn, S., Superfine, R., 2002. Torsional response and stiffening of individual multiwalled carbon nanotubes. Physical Review Letters 89, 255502.

Yakobson, B., Brabec, C., Bernholc, J., 1996. Nanomechanics of carbon tubes: Instabilities beyond the linear response. Physical Review Letters 76 (14), 2511-2514.

Yu, M., Lourie, O., Dyer, M., Moloni, K., Kelly, T., Ruoff, R., 2000. Strength and breaking mechanism of multiwalled carbon nanotubes under tensile load. Science 287, 637-640.

Zhang, M., Atkinson, K. R., Baughman, R. H., 2004. Multifunctional Carbon Nanotube Yarns by Downsizing an Ancient Technology. Science 306 (5700), $1358-1361$.

Zhang, S., Mielke, S., Khare, R., Troya, D., Ruoff, R., Schatz, G., Belytschko, T., 2005. Mechanics of defects in carbon nanotubes: Atomistic and multiscale simulations. Physical Review B 71 (11), 115403.

Zhang, S. L., Khare, R., Belytschko, T., Hsia, K. J., Mielke, S. L., Schatz, G. C., 2006. Transition states and minimum energy pathways for the collapse of carbon nanotubes. Physical Review B 73 (7), 075423.

Zhigilei, L., Wei, C., Srivastava, D., 2005. Mesoscopic model for dynamic simulations of carbon nanotubes. Physical Review B 71 (16), 165417. 


\section{COPYRIGHTED MATERIAL.}

Fig. 1. Experimental TEM observations of rippling deformations in bending: (a) Kuzumaki et al. (1998), (b,c) Bower et al. (1999), (d,e) Lourie et al. (1998), (f) Poncharal et al. (1999). 


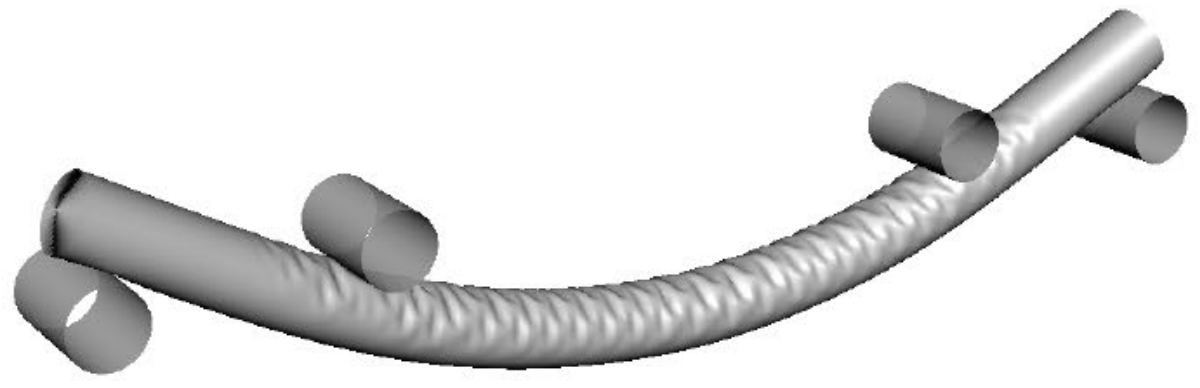

(a)

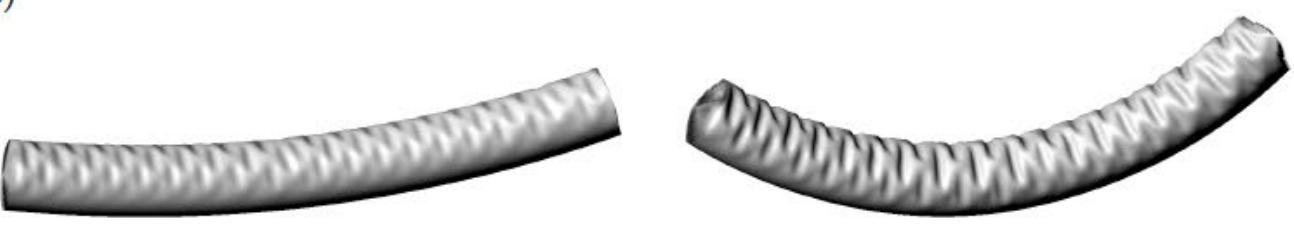

(b)

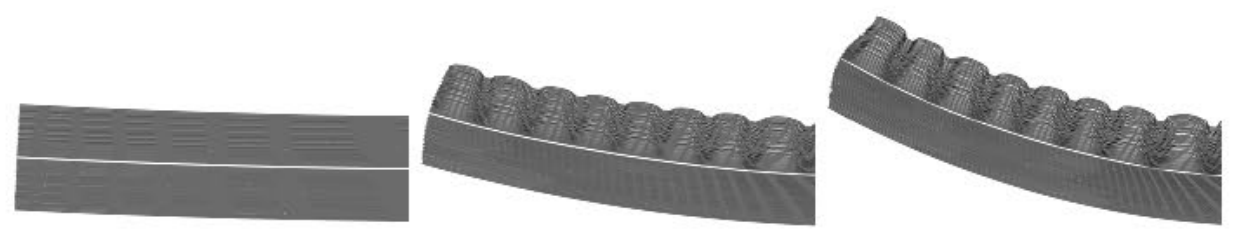

(c)
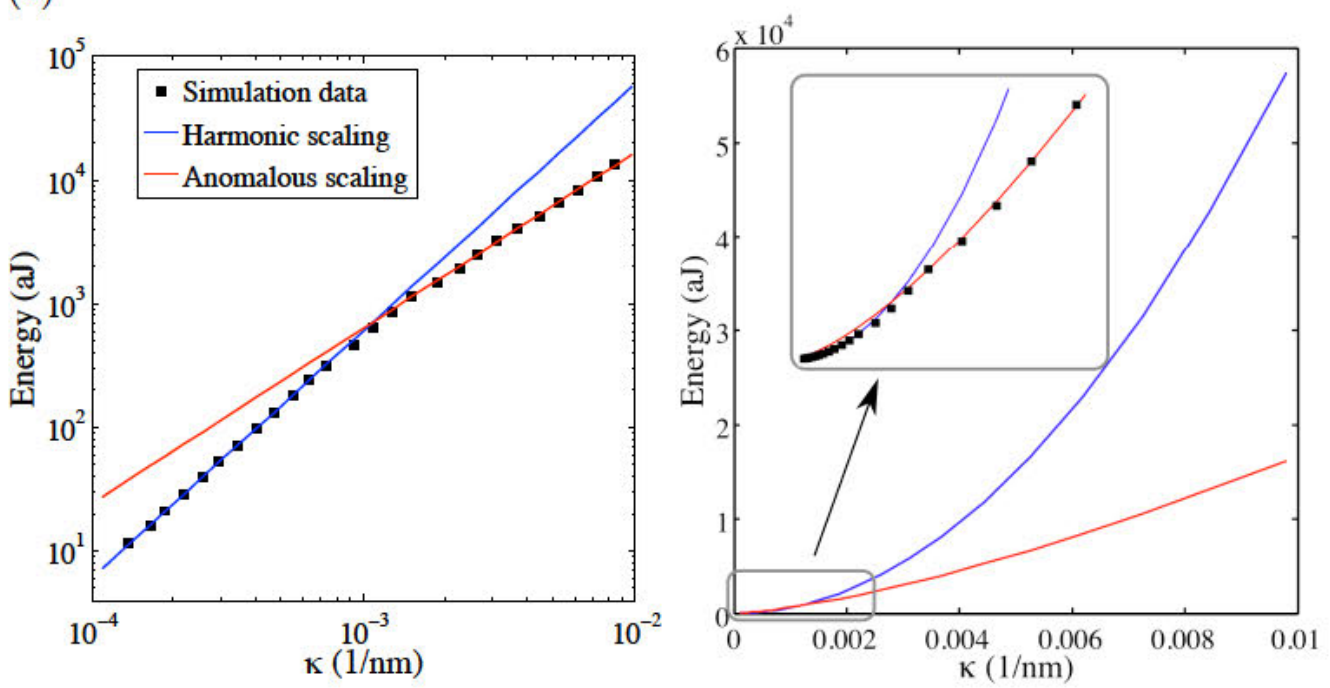

(d)

Fig. 2. (a) Simulation setup for the pure bending test of a 30 -walled CNT 360 $\mathrm{nm}$ long, (b) snapshots of the central uniformly bent part of the nanotube at two loading stages (the diamond deformation pattern is apparent), (c) cross-sections highlighting the importance of relative sliding of the walls, and (d) strain energy scaling vs. curvature in log-log scale (left) and in linear scale (right). 


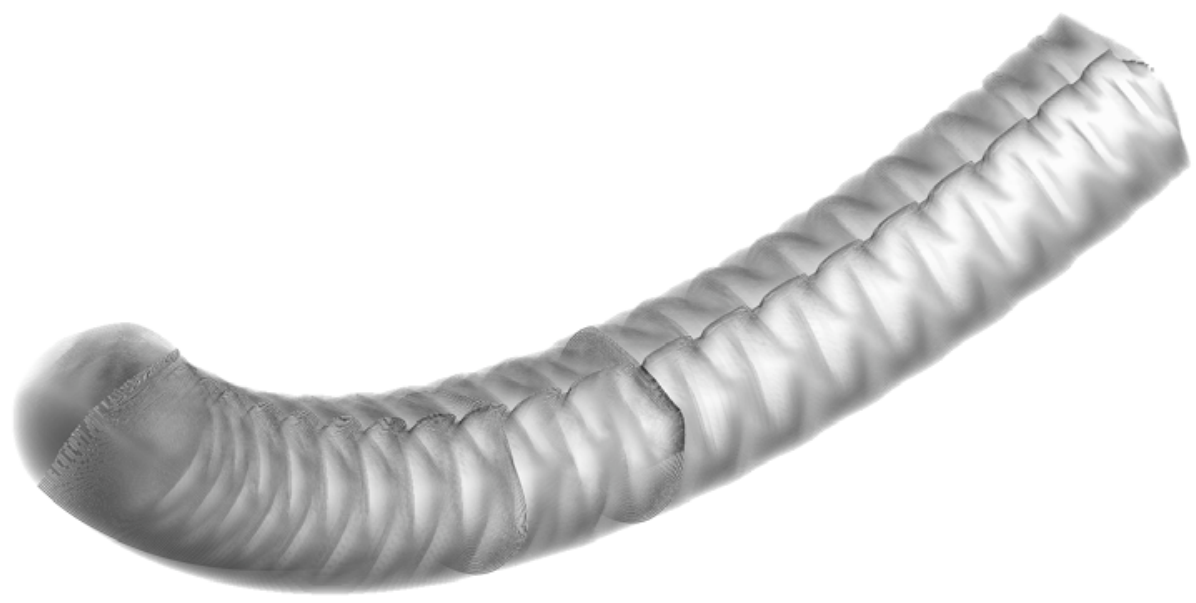

(a)

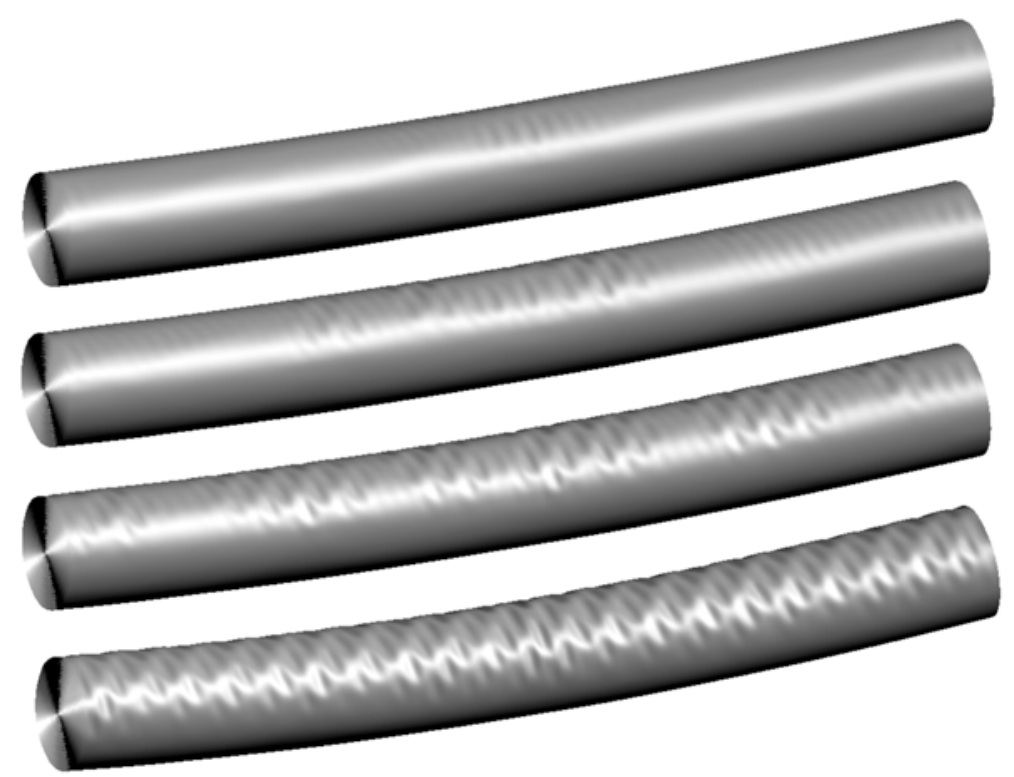

(b)

Fig. 3. Pure bending of a 40-walled CNT (only the central section $240 \mathrm{~nm}$ long is shown): (a) Visualization of the inner walls for a highly bent equilibrium configuration and (b) snapshots of four loading steps near the onset of rippling. Mixtures of a sinusoidal Fourier deformation mode of classical linearized buckling analysis and a Yoshimura or diamond pattern characteristic of post-buckling of thin cylindrical shells can be observed. The discrepancy between the linearized buckling and the postbuckling wavelengths is apparent. 


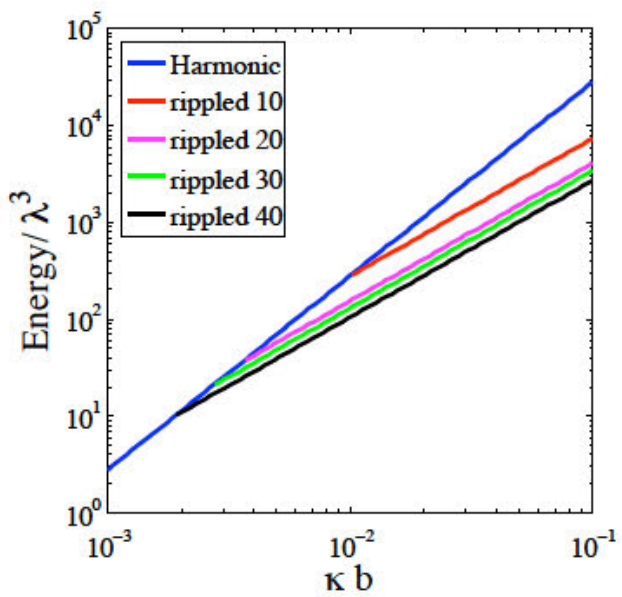

(a)

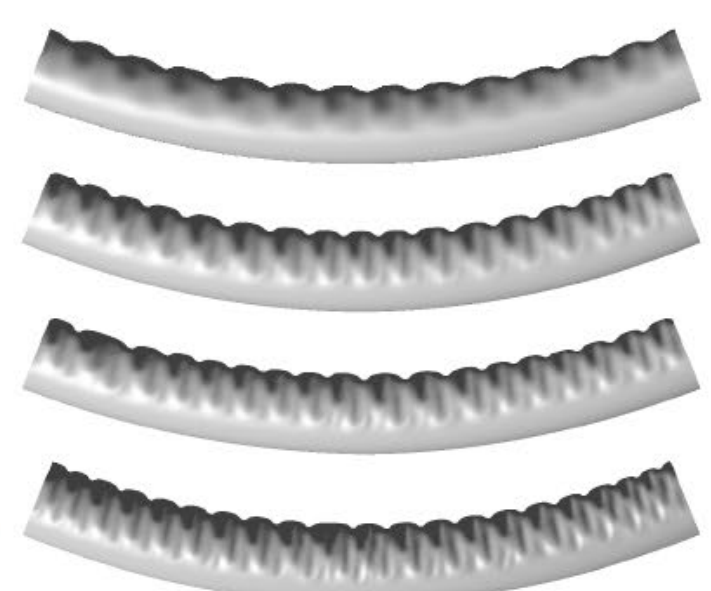

(b)

Fig. 4. Size effect in the bending response of MWCNTs: (a) Upon appropriate re-scaling, the curvature-energy relationships collapse for harmonic CNTs of different sizes, while the anharmonic response displays a strong size effect. (b) Outer shell of 10-walled, 20-walled, 30-walled and 40-walled CNTs subject to approximately the same non-dimensional curvature $\bar{\kappa}$. The rippled deformation is ostensibly not scale-invariant suggesting the size-effect. 


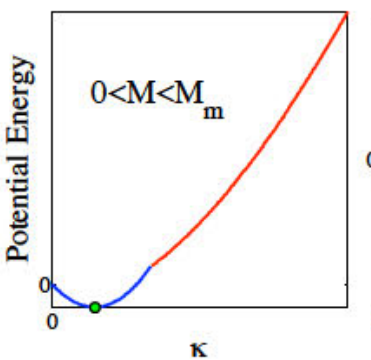

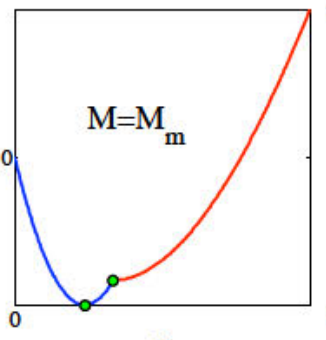

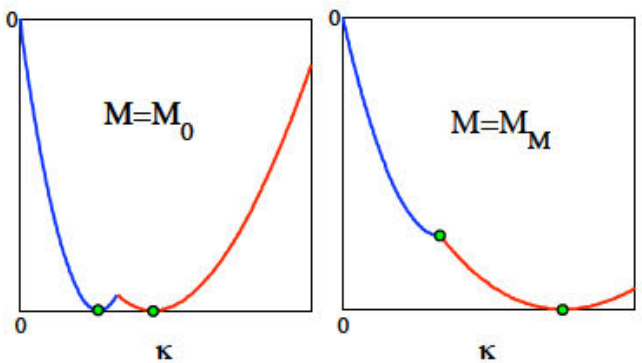

к

$\kappa$

Fig. 5. Emergence and destruction of energy minima upon loading for the non-convex energy. The potential energy minima representing the material stable states are represented by the green dots. 

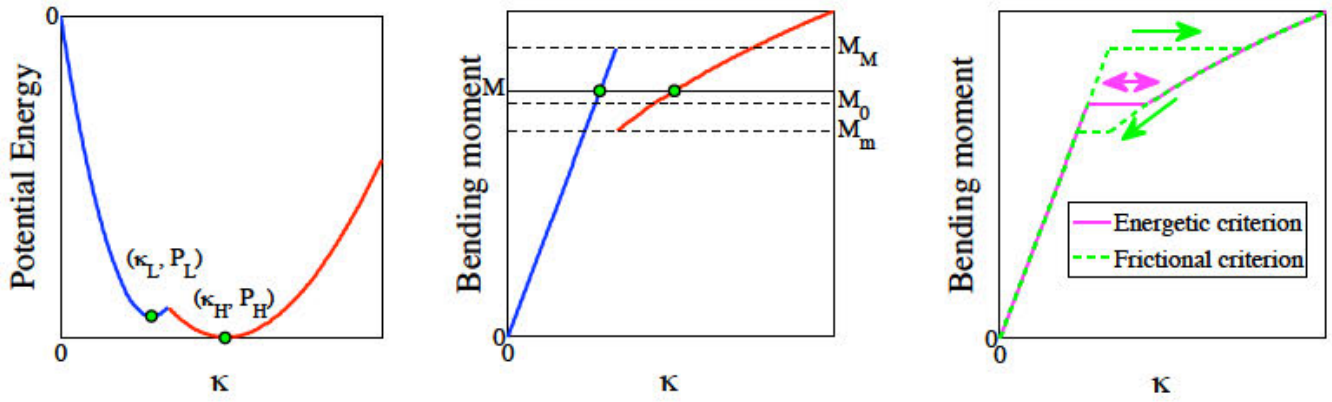

Fig. 6. Potential energy for a generic applied bending moment $M \in\left(M_{m}, M_{M}\right)$ (left), non-monotonic moment-curvature relation (center), and loading-unloading cycles for an elastica subjected to a uniform bending moment with a nucleation site at one end (right). 

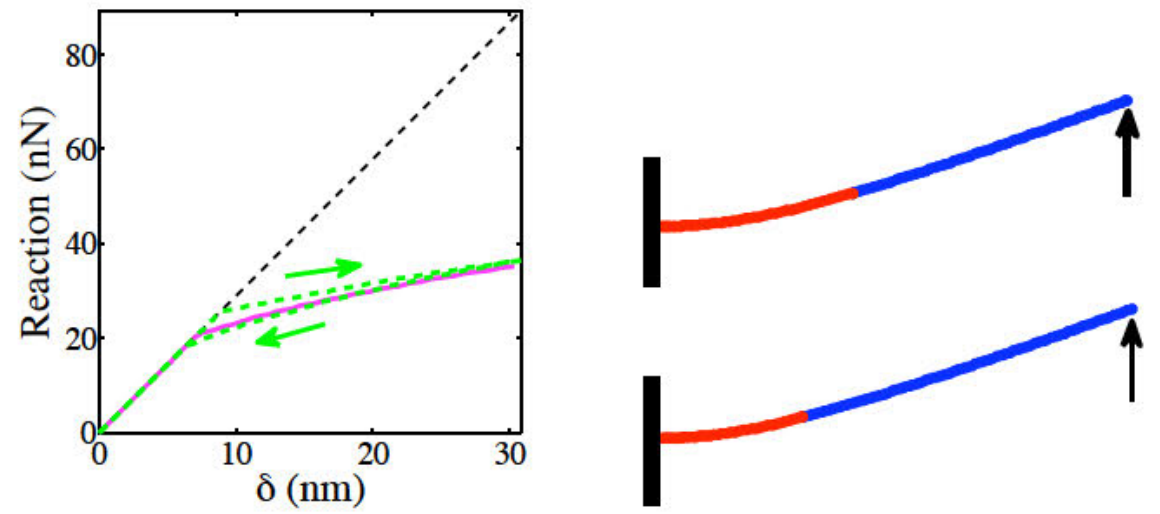

Fig. 7. Force-deflection response for a 20 -walled cantilevered CNT, and phase composition and deformation (not magnified) at the highest load for the energetic (top) and the frictional (bottom) criteria. The color coding (magenta for energetic model, green for frictional model; red for high-strain phases, blue for low-strain phase) is kept throughout the paper. 


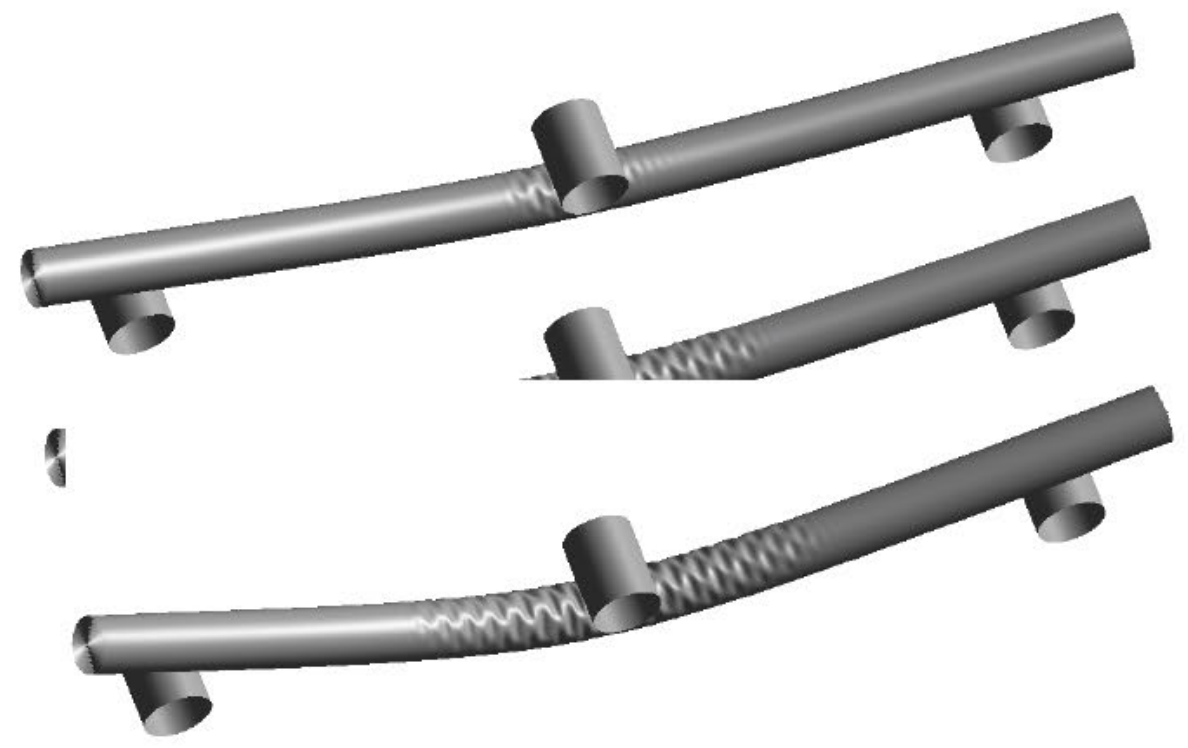

Fig. 8. View of the three dimensional deformation of a 20 -walled CNT $280 \mathrm{~nm}$ long subjected to a vertical displacement of $28 \mathrm{~nm}$, from the onset of rippling to the last computed configuration. 


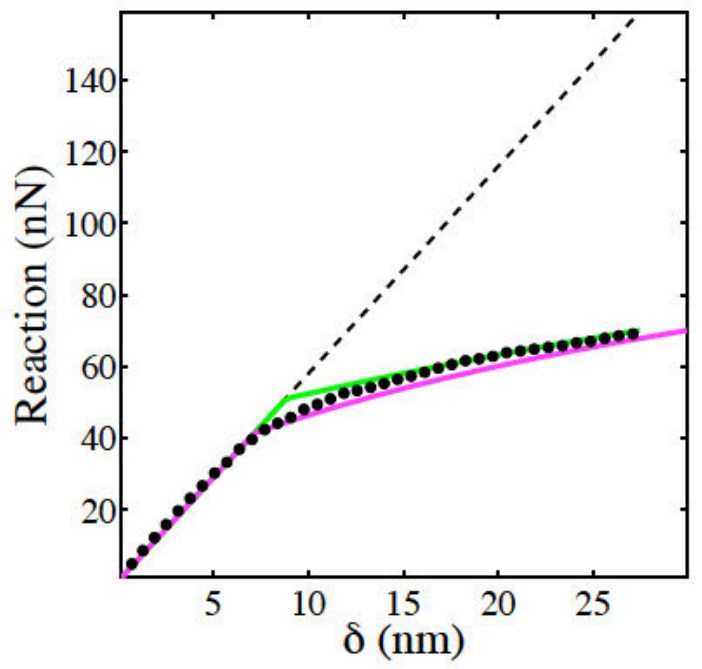

Fig. 9. Reaction force versus imposed vertical displacement for a linear elastic beam model (dashed), the mesoscopic beam model (frictional criterion in green, energetic criterion in magenta) and the 3D large-scale simulations (dots). 


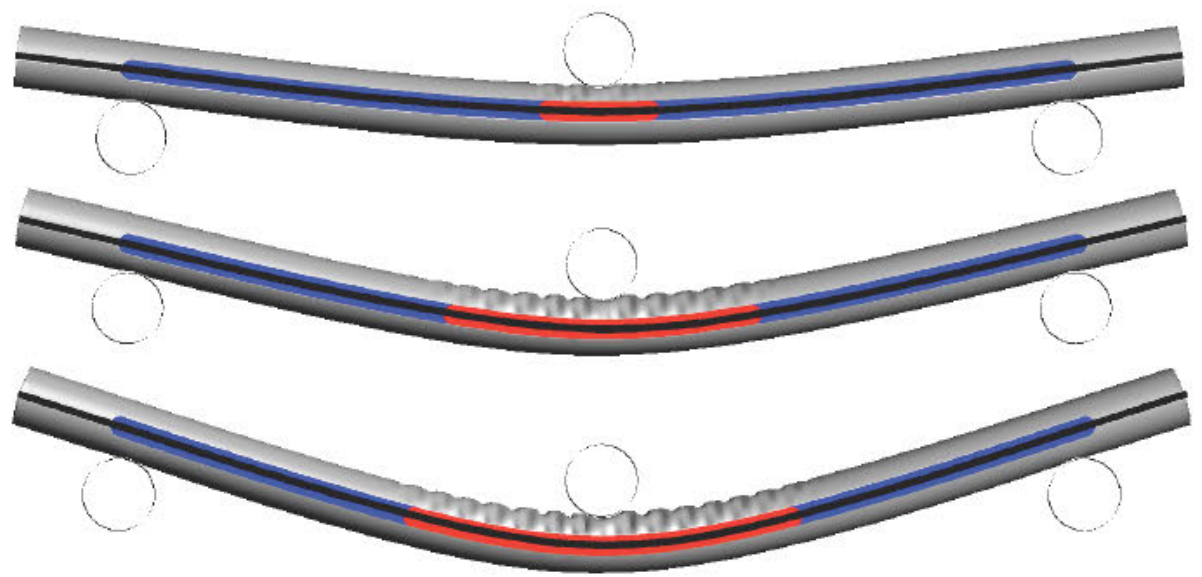

Fig. 10. Comparison between the deformations and rippling patterns provided by the full 3D model and the mesoscopic beam model with the energetic criterion for three selected loading stages. 
(a)

(b)

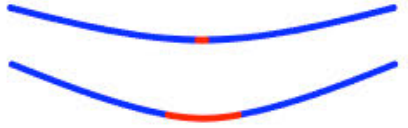

(c)

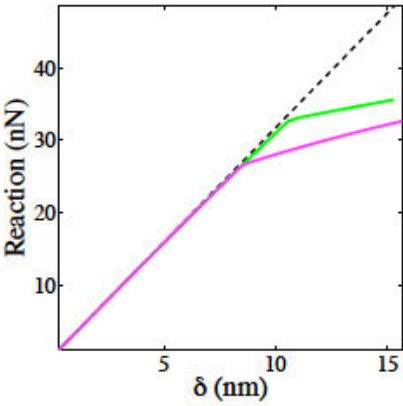

10-walled CNT 140 nm long
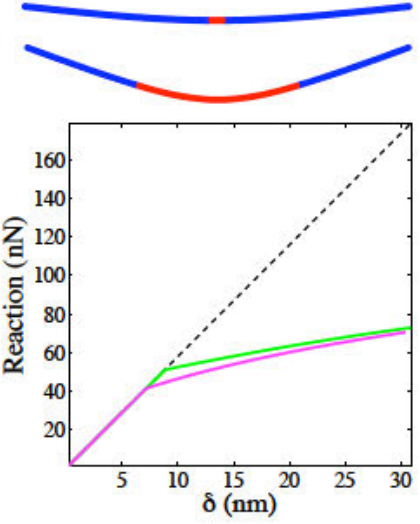

20-walled CNT $280 \mathrm{~nm}$ long
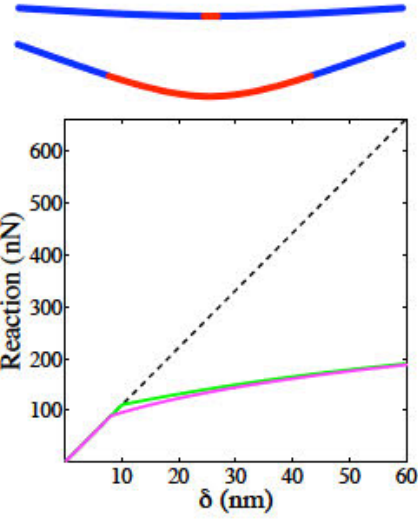

40-walled CNT $560 \mathrm{~nm}$ long

Fig. 11. Size effect in a three-point-bending test with self-similar geometry (constant length to diameter ratio and length to imposed displacement ratio): (a) Deformation at the nucleation of a rippled phase (in red), (b) deformation and phase distribution at the last stage (for the energetic criterion), and (c) force-displacement response for the energetic (magenta) and frictional (green) criteria and linearly elastic response $(--)$. 

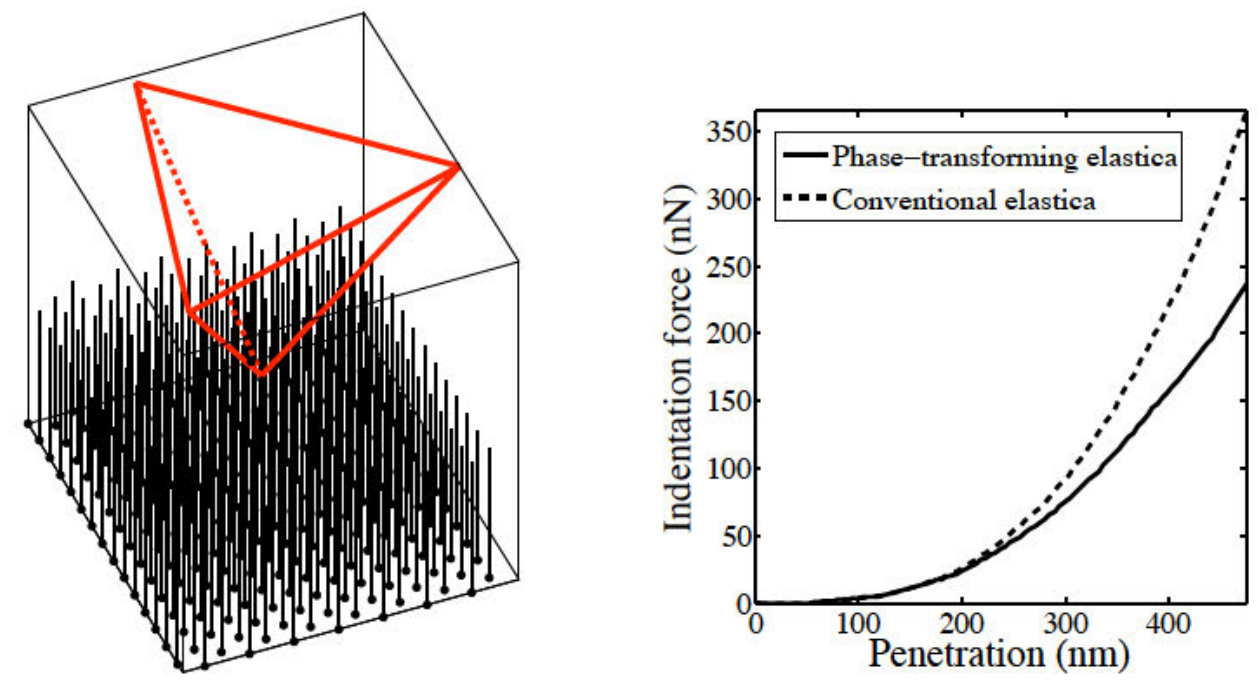

Fig. 12. Indentation of a forest of 40 -walled CNTs $500 \mathrm{~nm}$ long disposed in an hexagonal lattice with a density of 200 nanotubes per squared micron: setup of the forest and the AFM tip (left), and force-penetration curves obtained with the proposed model and a standard elastic beam model (right). 